Check for updates

1 Centre of General Practice, Department of Public Health, University of Copenhagen, Denmark

2 Primary Health Care Research Unit, Region Zealand, Denmark

3 Institute of Sports Medicine, Bispebjerg Hospital, Copenhagen, Denmark

Correspondence to: J Bülow jacob.buelow.02@regionh.dk

Cite this as: BMJ 2022;376:e052592 http://dx.doi.org/10.1136/bmj-2019-052592 Published: 13 January 2022

\title{
TOO MUCH MEDICINE
}

\section{Sarcopenia: early prevention or overdiagnosis?}

\section{Sarcopenia has recently been included in the international classification of diseases despite lack of evidence to support essential diagnostic aspects. Christoffer Bjerre Haase and colleagues argue that the change is a step towards overdiagnosis}

\section{Christoffer Bjerre Haase, ${ }^{1}$ John Brandt Brodersen, ${ }^{1,2}$ Jacob Bülow ${ }^{3}$}

\section{Summary box}

- Clinical context-Sarcopenia is defined as age related loss of muscle mass and function and is associated with increased morbidity and mortality. With rising numbers of older people, interest in the condition and possible treatments is expected to rise

- Diagnostic change-Sarcopenia was first described in 1989 as the phenomenon of decreasing lean body mass with older age. The idea of sarcopenia as a disease was raised in 1997. From 2010 to 2014, six consensus definitions changed the focus to assessments of physical function. In 2016, sarcopenia was assigned the code M62.84 in the International Classification of Diseases (ICD-10-CM)

- Rationale for change-Sarcopenia specialists' groups argued that an ICD-10 diagnosis would raise awareness and recognition of the condition, encourage funders and sponsors to allocate research resources, and support development of new therapies

- Leap of faith-Early detection and treatment of sarcopenia will reduce morbidity and mortality and improve quality of life

- Effect on prevalence-Based on the most used definition of sarcopenia, prevalence is estimated at 5-13\% among people aged $60-70$ years, and $11-50 \%$ among people aged $>80$ years. Worldwide prevalence by 2050 could be up to two billion

- Evidence of overdiagnosis-Current literature, including studies on screening for sarcopenia, has not explicitly considered the risk of overdiagnosis. For now, overdiagnosis is inevitable since treatment does not differ from general health recommendations

- Harms from overdiagnosis-No studies have investigated how people are affected by being diagnosed with sarcopenia. Indirect evidence shows that being labelled with a diagnosis that implies increased risk of morbidity and mortality imposes a psychological burden

- Limitations of evidence-A diagnosis of sarcopenia has not been shown to improve prognosis. Sarcopenia treatment has not been shown to have better outcomes than general recommendations for physical exercise and diet. Moreover, the current diagnostic cut-off points, including sex and regional adjustments, are arbitrary and non-validated. It is not possible to distinguish between normal and pathological age related loss of muscle mass

As the global population ages, science and societies look for initiatives to handle the societal and individual problems that follow. One such initiative is the diagnosis of sarcopenia, the phenomenon of age related loss of muscle mass and function. Around two billion people aged 60 years or older are expected to be diagnosed with sarcopenia by $2050 .{ }^{1}$ Yet despite important research, uncertainties about the clinical value of diagnosis remain. We examine how modern medicine has established the diagnosis of sarcopenia without sufficient supporting evidence and ask whether it will lead to better prevention and treatment or to overdiagnosis.

\section{Criteria for diagnosis}

Guidance from European and international specialist groups suggests a diagnosis of sarcopenia is probable when patients are found to have low muscle strength measured by either grip strength or chair stand test. Sarcopenia is confirmed when this is combined with "the presence of low muscle quantity or quality" measured by dual energy $\mathrm{x}$ ray absorptiometry, bioelectrical impedance analysis, muscle biopsies, computed tomography, or magnetic resonance imaging (table 1$){ }^{2}$ When patients also have poor physical performance, sarcopenia is considered severe. ${ }^{2}$ The diagnostic cut-off point is given as 2-2.5 standard deviations below the mean of a sex and regional specific reference population of healthy young adults. ${ }^{2}$ When recording the diagnosis, any underlying disease should be coded first. If none exists, sarcopenia should be coded before associated conditions such as generalised weakness. 45 
Table 1 | Definitions of sarcopenia and estimates of prevalence

\begin{tabular}{|c|c|c|c|}
\hline Author & Description & Definition & Prevalence \\
\hline $\begin{array}{l}2010 \text { European Working Group on Sarcopenia } \\
\text { in Older People } 1\end{array}$ & $\begin{array}{l}\text { "Sarcopenia is a syndrome characterised by } \\
\text { progressive and generalised loss of skeletal } \\
\text { muscle mass and strength with a risk of } \\
\text { adverse outcomes such as physical disability, } \\
\text { poor quality of life and death" }\end{array}$ & $\begin{array}{l}\text { Diagnosis is based on documentation of low } \\
\text { muscle mass plus low muscle strength or low } \\
\text { physical performance }\end{array}$ & $\begin{array}{l}60-70 \text { year old: } 5-13 \% \text {, } \\
\text { > } 80 \text { years old: } 11-50 \% \text {, } \\
\geq 60 \text { years old, worldwide: } \\
600 \text { million in } 2000,1.2 \text { billion in } 2025 \text {, and } 2 \\
\text { billion in } 2050 \text {. } \\
\text { (Conservative estimate }>50 \text { million people } \\
\text { today, > } 200 \text { million in the next } 40 \text { years) }\end{array}$ \\
\hline $\begin{array}{l}2018 \text { European Working Group on Sarcopenia } \\
\text { in Older People (EWGSOP2) }\end{array}$ & $\begin{array}{l}\text { "Sarcopenia is a progressive and generalised } \\
\text { skeletal muscle disorder that is associated with } \\
\text { increased likelihood of adverse outcomes } \\
\text { including falls, fractures, physical disability and } \\
\text { mortality" }\end{array}$ & $\begin{array}{l}\text { "Sarcopenia is now defined as a muscle disease } \\
\text { that may be acute or chronic." } \\
\text { Criteria: 1. Low muscle strength; } 2 \text {. Low muscle } \\
\text { quantity or quality; 3. Low physical } \\
\text { performance } \\
\text { Probable sarcopenia is identified by criterion } 1 \\
\text { Diagnosis is confirmed by additional } \\
\text { documentation of criterion } 2 \\
\text { If all three criteria are met, sarcopenia is } \\
\text { considered severe }\end{array}$ & Not stated \\
\hline $\begin{array}{l}\text { International Clinical Practice Guidelines for } \\
\text { Sarcopenia (ICSFR), } 2018^{3}\end{array}$ & $\begin{array}{l}\text { "Sarcopenia is defined as an age-associated } \\
\text { loss of skeletal muscle function and muscle } \\
\text { mass, and is common in older adults ... The } \\
\text { most commonly used diagnostic tool is that of } \\
\text { the EWGSOP" }\end{array}$ & & $\begin{array}{l}6-22 \% \text { adults aged } \geq 65 \text { years with a variation } \\
\text { in prevalence across healthcare settings }\end{array}$ \\
\hline
\end{tabular}

Sarcopenia specialist groups consider sarcopenia to be the most important cause of frailty in older people. ${ }^{6}$ It is positively correlated with multiple health related conditions, including falls and fractures, cardiac and respiratory diseases, cognitive impairment, low quality of life, and death. The condition is costly because of increased hospital admission and an associated increased need for care while in hospital. ${ }^{2}$

Sarcopenia is common in older people but can also affect younger people. Ageing is the cause of primary sarcopenia. Secondary sarcopenia can have multiple causes, including lack of activity, age related decline in testosterone, genetic factors, and insufficient energy or protein intake because of anorexia or malabsorption. ${ }^{67}$ Sarcopenia lasting for at least six months is considered chronic. ${ }^{2}$

Depending on definition and the investigated population, the prevalence varies from $5 \%$ to $50 \%$ of people $\geq 60$ years old (table 1). ${ }^{1-3689}$ The international clinical practice guideline for sarcopenia (ICFSR) recommends annual screening of everyone older than 65 in general practice or outpatient clinics using a tool such as SARC-F (box 1)..$^{23}$

\section{Box 1: SARC-F (strength, assistance walking, rise from a chair, climb} stairs, and falls) screening tool for sarcopenia ${ }^{10}$

\section{Questions}

- How much difficulty do you have in lifting and carrying 10 pounds?

- $($ None $=0$, some $=1$, A lot or unable $=2$ )

- How much difficulty do you have transferring from a chair or bed?

- $\quad$ (None $=0$, some =1, A lot or unable without help =2)

- How much difficulty do you have walking across a room?

- $\quad$ (None $=0$, some $=1$, A lot, use aids, or unable $=2$ )

- How much difficulty do you have climbing a flight of ten stairs?

- $($ None $=0$, some $=1$, A lot or unable $=2$ )

- How many times have you fallen in the last year?

- $($ None $=0,1-3$ falls $=1,4$ or more falls $=2$ )

Sarcopenia is diagnosed if total score is $\geq 4$

\section{Performance data}

- The test has sensitivity of 0.21 ( $95 \% \mathrm{Cl} 0.13$ to 0.31$)$, specificity of 0.90 (0.83 to 0.94 ), positive likelihood ratio of 2.16 (1.51 to 3.09), and negative likelihood ratio of 0.87 (0.80 to 0.95$).{ }^{11}$ Diagnostic odds ratio is 2.47 (1.64 to 3.74$).{ }^{11}$

- The strength of evidence is rated as conditional with a low certainty and the tool has not been assessed against WHO's 10 screening principles or for risk of overdiagnosis. ${ }^{2} 511-13$

- Current guidelines recommend the SARC-F for clinical use. ${ }^{23}$

Treatment for sarcopenia is currently supported by limited evidence and consists of resistance exercise, optionally supplemented with a high intake of essential amino acids and vitamin D. ${ }^{2367}$ Testosterone has been suggested as a potential treatment and new drugs are under development, such as myostatin inhibitors. ${ }^{79}$

\section{Rationale for change}

In 1989, medical doctor and researcher in nutrition Irwin $\mathrm{H}$ Rosenberg introduced sarcopenia as a term to define and articulate the natural phenomenon of loss of skeletal muscle mass with age. ${ }^{14}$ Eight years later, Rosenberg questioned whether sarcopenia could be defined as a disease. ${ }^{15}$ During 2010 to 2014, six consensus definitions were agreed. ${ }^{116-20}$ Each definition positioned sarcopenia as a disease, without reference to Rosenberg's concern. Instead, they focused on making sarcopenia more relevant for clinicians and patients by reorienting the definition towards muscle function rather than muscle mass, since muscle function and strength were more strongly correlated with clinically relevant outcomes, such as morbidity and mortality. ${ }^{116-20}$

An ICD-10 diagnosis was proposed in 2014 to "raise awareness" and "reduce treatment barriers." ${ }^{21}$ Proponents argued that disease status would encourage drug companies to develop drugs ${ }^{6}$ and incentivise research, just as it did for osteoporosis. ${ }^{5}$ Sarcopenia officially became a diagnosis in 2016 with an international classification of diseases clinical modification code (ICD-10-CM) M62.84. ${ }^{24-7}$ Since 
then, supporters have been working for the creation of a unique code for the ICD-11.

\section{Uncertainty of evidence}

Everyone will experience muscle loss during their life, and some will experience greater loss than others. Nevertheless, the evidence to justify and specify the diagnosis in ICFSR is uncertain or missing (box 2), and the guideline admits: "There exists considerable room for improvement of the methodological quality of clinical trials for sarcopenia. The quality of supporting evidence for the management of sarcopenia was low." ${ }^{3}$ The following three questions remain unanswered.

Box 2: Current uncertainties in diagnosis of sarcopenia $2522-25$ Clinical practice

- No studies have shown any difference in treatment or prognosis following diagnosis

- Cut-off points for diagnosis are arbitrary

- Cut-off points for diagnosis are non-validated

- Cut-off points for gender and some regional specifications are missing

- It is unknown how to define muscle quality

- It is unknown which muscle quality indicators best predict relevant (clinical) outcomes

- It is unknown which outcomes are suitable for measuring intervention response ${ }^{2}$

- Studies are required to understand outcomes relevant and important to patients

- Studies are needed to investigate differences between primary and secondary sarcopenia ${ }^{3}$

- The strength of evidence for screening is classified as conditional with a low certainty of evidence. ${ }^{3}$ The 10 principles for screening, defined by $\mathrm{WHO},{ }^{26}$ have not been used to assess the screening tool $^{2} 511-13$

- No studies have investigated the potential harms of being labelled with the diagnosis of sarcopenia

- No studies have assessed the risk of overdiagnosis.

\section{Research}

- Various definitions of sarcopenia are still in use, including the original, dating back to $1989^{527}$

- The variation of different primary outcomes used in sarcopenia interventions has been described as "extreme" 5 by sarcopenia researchers. One study of 123 interventions found that less than $30 \%$ of the interventions measured muscle mass and strength as primary outcome 5

- Recruiting participants who actually match the criteria of sarcopenia has been described as a "major challenge" 5 by sarcopenia researchers

- Future clinical trials are recommended to include the actual target population. 3

\section{How is disease distinguished from normal age related changes?}

Diagnostic cut-off points, including sex and regional adjustments, are currently arbitrary and non-validated. In addition, it is unclear which muscle quality indicators best predict relevant clinical outcomes or how best to measure response to interventions. ${ }^{2}$ Sarcopenia researchers have described it as a "major challenge"5 to recruit research participants who match the criteria for primary sarcopenia. This suggests a lack of diagnostic clarity and may make it difficult to obtain robust high quality evidence (box 2).

\section{Does the diagnosis affect prognosis or treatment?}

Evidence is lacking that patients who are diagnosed with sarcopenia have improved outcomes. Nor has sarcopenia treatment been shown to produce better outcomes than general recommendations for physical exercise and diet (see supplementary data on bmj.com)..$^{22-25}$

Furthermore, ICFSR found "very low certainty for the beneficial effects of resistance based training in adults with sarcopenia" ${ }^{3}$ and low certainty regarding the evidence on protein supplementation. The safety and efficiency of medical treatments such as vitamin D, hormones, or creatinine are unknown because of inadequate data in people with sarcopenia, ${ }^{39}$ and they are not recommended as first line treatment. For vitamin D, the guideline also notes that because of "the ambiguity of results and low sample size of the majority of clinical trials on sarcopenia, there is a significant probability that health benefits may not outweigh potential undesirable outcomes.”

The World Health Organization already recommends that all people should be physically active, the positive effects of which are similar to those described for sarcopenia..$^{28}$ In 2002, WHO investigated sarcopenia, concluding that muscle strength can be increased with resistance exercise and a protein intake of $0.8 \mathrm{~g} / \mathrm{kg} /$ day, which is lower than the average intake of the elderly people included in sarcopenia studies. ${ }^{29} 30$

\section{Does the diagnosis cause unintended effects?}

To our knowledge, no studies have investigated the potential unintended effects or harms of being diagnosed with sarcopenia. It is therefore not possible to assess the balance of benefits and harms, an essential part of the process of recommending a new disease. . $^{31}$-33 ICFSR uses the GRADE system to evaluate evidence but assesses undesirable outcomes only in relation to vitamin D supplementation, stating that a "major concern is the lack of robust, large scale clinical trials with long term follow-up for older adults with sarcopenia." 3

\section{Risk of overdiagnosis}

The decision to classify a phenomenon as a disease involves a delicate balance between several factors, among which the benefit-to-harm ratio and the ability to separate normality from pathology are essential. When this balance is skewed, overdiagnosis is likely to occur.

Overdiagnosis is broadly defined as "making people patients unnecessarily, by identifying problems that were never going to cause harm or by medicalising ordinary life experiences through expanded definitions of diseases." ${ }^{34}$ It occurs across all medical disciplines and is a harmful and costly global problem in modern healthcare. ${ }^{33-36}$ No studies have investigated the possibility of overdiagnosing sarcopenia.

Proponents of classifying sarcopenia as a disease have compared its benefit to that of a diagnosis of osteoporosis and hypertension: "as a means to avoid disability." This comparison is questionable and assumes that these conditions are comparable-for example, that severe harms could occur if sarcopenia is left undiagnosed and untreated, such as fractures in osteoporosis and stroke and cardiovascular events in hypertension. Moreover, a more complete understanding of sarcopenia requires consideration of the potential harms known to occur from overdiagnosis of osteoporosis and hypertension. These include increased absenteeism from work, lower self-rated health, and psychological and relationship harm from, for example, anxiety and depression. ${ }^{37-43}$ 


\section{Early prevention or overdiagnosis?}

Research into age related loss of muscle mass is undoubtedly important and will become even more valuable in the future as the population ages. From this perspective, research into establishing sarcopenia as a disease is reasonable. Benefits such as medical treatment and easier access to help from social care systems and health insurance providers could follow a diagnosis. In addition, a diagnosis might motivate patients to pursue a healthier lifestyle. Thus, establishing sarcopenia as a disease may come to be seen as a great medical achievement.

However, as essential diagnostic questions remain unanswered, diagnosing sarcopenia in clinic deserves further consideration. If sarcopenia is considered a disease, the current uncertainties among researchers will be passed on to clinicians, and eventually patients. Clinicians accountable for patients need to be comfortable with the justification of the disease status and the evidence that underpins it.

\section{How to do better}

Before establishing sarcopenia as a disease we need evidence that it meets essential diagnostic criteria. From a medical perspective, clinically assessing and improving the health of a vulnerable patient does not necessarily require more diagnoses than those already available.

Muscle wasting has long been recognised as a problem in the elderly population, but until medical science provides evidence in favour of a diagnostic category, we should look at sarcopenia as Rosenberg originally did-as a natural phenomenon of age related loss of muscle mass. A broader view may also support the creation of social, economic, psychological, and educational initiatives that consider not only the biomedical but also the social, psychological, and existential problems of growing older.

Contributors and sources: $\mathrm{CBH}$ is a doctor with a special interest in data driven diagnostics in relation to overdiagnosis. JBB has international expertise within overdiagnosis, and JB has studied muscle physiology with a special focus on age related changes and prevention strategies. JB got the idea for the project during his $\mathrm{PhD}$, and the article arose from a discussion at a meeting on how diagnoses are established. All three authors contributed to the conception, the design, the acquisition, analysis, and interpretation of the work. CBH and JB drafted the first version of the manuscript. All authors revised it critically for important intellectual content and approved the final version.

Competing interests: We have read and understood BMJ policy on declaration of interests and have no relevant interests to declare.

Provenance and peer review: Not commissioned; externally peer reviewed.

We thank Steven Woloshin, Dartmouth Institute and the Lisa Schwartz Foundation for Truth in Medicine, for comments on an early draft.

Cruz-Jentoft AJ, Baeyens JP, Bauer JM, etalEuropean Working Group on Sarcopenia in Older People. Sarcopenia: European consensus on definition and diagnosis: Report of the European Working Group on Sarcopenia in Older People. Age Ageing 2010;39:412-23.

doi: 10.1093/ageing/afq034 pmid: 20392703

2 Cruz-Jentoft AJ, Bahat G, Bauer J, etal. Writing Group for the European Working Group on Sarcopenia in Older People 2 (EWGSOP2), and the Extended Group for EWGSOP2. Sarcopenia: revised European consensus on definition and diagnosis. Age Ageing 2019;48:16-31. doi: 10.1093/ageing/afy169 pmid: 30312372

3 Dent E, Morley JE, Cruz-Jentoft AJ, etal. International clinical practice guidelines for sarcopenia (ICFSR): screening, diagnosis and management. J Nutr Health Aging 2018;22:1148-61. doi: 10.1007/s12603-018-1139-9 pmid: 30498820

42019 ICD-10-CM Diagnosis Code M62.84: Sarcopenia. https://www.icd10data.com/ICD10CM/Codes/M00-M99/M60-M63/M62-/M62.84

5 Vellas B, Fielding RA, Bens C, etal. Implications of ICD-10 for sarcopenia clinical practice and clinical trials: report by the international conference on frailty and sarcopenia research task force. J Frailty Aging 2018;7:2-9. doi: 10.14283/jfa.2017.30.. pmid: 29412436

6 Anker SD, Morley JE, von Haehling S. Welcome to the ICD-10 code for sarcopenia. J Cachexia Sarcopenia Muscle 2016;7:512-4. doi: 10.1002/jcsm.12147 pmid: 27891296
7 Cao L, Morley JE. Sarcopenia is recognized as an independent condition by an International Classification of Disease, Tenth Revision, Clinical Modification (ICD-10-CM) Code. J Am Med Dir Assoc 2016;17:675-7. doi: 10.1016/j.jamda.2016.06.001 pmid: 27470918

8 Ethgen O, Beaudart C, Buckinx F, Bruyère O, Reginster JY. The future prevalence of sarcopenia in Europe: a claim for public health action. Calcif Tissue Int 2017;100:229-34. doi: 10.1007/s00223-016-0220-9 pmid: 28012107

9 Rolland Y, Onder G, Morley JE, Gillette-Guyonet S, Abellan van Kan G, Vellas B. Current and future pharmacologic treatment of sarcopenia. Clin Geriatr Med 2011;27:423-47. doi: 10.1016/j.cger.2011.03.008 pmid: 21824556

10 Morley JE, Cao L. Rapid screening for sarcopenia. J Cachexia Sarcopenia Muscle 2015;6:312-4. doi: 10.1002/jcsm.12079 pmid: 26676168

11 Ida S, Kaneko R, Murata K. SARC-F for screening of sarcopenia among older adults: a meta-analysis of screening test accuracy. J Am Med Dir Assoc 2018;19:685-9.

doi: 10.1016/j.jamda.2018.04.001 pmid: 29778639

12 Bahat G, Yilmaz O, Kilıç C, Oren MM, Karan MA. Performance of SARC-F in regard to sarcopenia definitions, muscle mass and functional measures. J Nutr Health Aging 2018;22:898-903. doi: 10.1007/s12603-018-1067-8 pmid: 30272090

13 Bahat G, Yilmaz O, Oren MM, etal. Cross-cultural adaptation and validation of the SARC-F to assess sarcopenia: methodological report from European Union Geriatric Medicine Society Sarcopenia Special Interest Group. Eur Geriatr Med 2018;9:23-8. doi: 10.1007/s41999-017-0003-5 pmid: 34654275

14 Rosenberg IH. Summary comments. Am J Clin Nutr 1989;50:1231-3. doi: 10.1093/ajcn/50.5.1231

15 Rosenberg IH. Sarcopenia: origins and clinical relevance. J Nutr 1997;127(Suppl):990S-1S. doi: 10.1093/jn/127.5.990S pmid: 9164280

16 Chen L-K, Liu L-K, Woo J, etal. Sarcopenia in Asia: consensus report of the Asian Working Group for Sarcopenia. J Am Med Dir Assoc 2014;15:95-101. doi: 10.1016/j.jamda.2013.11.025 pmid: 24461239

17 Fielding RA, Vellas B, Evans WJ, etallnternational Working Group on Sarcopenia. Sarcopenia: an undiagnosed condition in older adults. Current consensus definition: prevalence, etiology, and consequences. International working group on sarcopenia. J Am Med Dir Assoc 2011;12:249-56 doi: 10.1016/j.jamda.2011.01.003 pmid: 21527165

18 Morley JE, Abbatecola AM, Argiles JM, etalSociety on Sarcopenia, Cachexia and Wasting Disorders Trialist Workshop. Sarcopenia with limited mobility: an international consensus. J Am Med Dir Assoc 2011;12:403-9. doi: 10.1016/j.jamda.2011.04.014 pmid: 21640657

19 Muscaritoli M, Anker SD, Argilés J, etal. Consensus definition of sarcopenia, cachexia and pre-cachexia: joint document elaborated by Special Interest Groups (SIG) "cachexia-anorexia in chronic wasting diseases" and "nutrition in geriatrics." Clin Nutr 2010;29:154-9. doi: 10.1016/j.clnu.2009.12.004 pmid: 20060626

20 Studenski SA, Peters KW, Alley DE, etal. The FNIH sarcopenia project: rationale, study description, conference recommendations, and final estimates. J Gerontol A Biol Sci Med Sci2014;69:547-58 doi: 10.1093/gerona/glu010 pmid: 24737557

21 Lloyd N. AIM coalition announces establishment of ICD-10-CM code for sarcopenia by the Centers for Disease Control and Prevention. Aging in Motion 2016. http://aginginmotion.org/news/2388 2/

22 Bloom I, Shand C, Cooper C, Robinson S, Baird J. Diet quality and sarcopenia in older adults: a systematic review. Nutrients 2018;10:308. doi: 10.3390/nu10030308 pmid: 29510572

23 Steffl M, Bohannon RW, Sontakova L, Tufano JJ, Shiells K, Holmerova I. Relationship between sarcopenia and physical activity in older people: a systematic review and meta-analysis. Clin Interv Aging 2017;12:835-45. doi: 10.2147/CIA.S132940 pmid: 28553092

24 Cederholm T. Overlaps between frailty and sarcopenia definitions. In: Nestle Nutrition Institute workshop series. Karger Publishers, 2015: 65-70. doi: 10.1159/000382063

25 Dodds R, Sayer AA. Sarcopenia and frailty: new challenges for clinical practice. Clin Med (Lond) 2015;15(Suppl 6):s88-91. doi: 10.7861/clinmedicine.15-6-s88 pmid: 26634689

26 Wilson J, Jungner G. Principles and practice of screening for disease. WHO Public Paper 34. 1968 https://apps.who.int/iris/bitstream/handle/10665/37650/WHO_PHP_34.pdf?sequence=17\&isAllowed=y

27 Larsson L, Degens H, Li M, etal. Sarcopenia: aging-related loss of muscle mass and function. Physiol Rev 2019;99:427-511. doi: 10.1152/physrev.00061.2017 pmid: 30427277

28 World Health Organization. Global recommendations on physical activity for health. WHO, 2010.

29 Protein and amino acid requirements in human nutrition: report of a joint FAO/WHO/UNU expert consultation. WHO, 2002

30 Campbell WW, Trappe TA, Jozsi AC, Kruskall LJ, Wolfe RR, Evans WJ. Dietary protein adequacy and lower body versus whole body resistive training in older humans. J Physiol 2002;542:631-42. doi: 10.1113/jphysiol.2002.020685 pmid: 12122158

31 Guyatt GH, Oxman AD, Vist GE, etalGRADE Working Group. GRADE: an emerging consensus on rating quality of evidence and strength of recommendations. BMJ 2008;336:924-6. doi: 10.1136/bmj.39489.470347.AD.. pmid: 18436948

32 Guyatt G, Rennie D, Meade M, etal. Users' guides to the medical literature : a manual for evidence-based clinical practice. 3rd ed. McGraw-Hill Education, 2015, https://jamaevidence. mhmedical.com/content.aspx?bookid=847\&sectionid=69030714.

33 Doust J, Vandvik PO, Qaseem A, etalGuidelines International Network (G-I-N) Preventing Overdiagnosis Working Group. Guidance for modifying the definition of diseases: A checklist. JAMA Intern Med 2017;177:1020-5. doi: 10.1001/jamainternmed.2017.1302 pmid: 28505266 
34 Brodersen J, Schwartz LM, Heneghan C, O’Sullivan JW, Aronson JK, Woloshin S. Overdiagnosis: what it is and what it isn't. BMJ Evid Based Med 2018;23:1-3. doi: 10.1136/ebmed-2017-110886. pmid: 29367314

35 Moynihan R, Brodersen J, Heath I, etal. Reforming disease definitions: a new primary care led, people-centred approach. BMJ Evid Based Med 2019;24:170-3.pmid: 30962252

36 Brodersen J, Kramer BS, Macdonald H, Schwartz LM, Woloshin S. Focusing on overdiagnosis as a driver of too much medicine. BM/2018;362:k3494. doi: 10.1136/bmj.k3494 pmid: 30120097

37 Järvinen TLN, Michaëlsson K, Jokihaara J, etal. Overdiagnosis of bone fragility in the quest to prevent hip fracture. BMJ2015;350:h2088. doi: 10.1136/bmj.h2088 pmid: 26013536

38 Alderman MH, Lamport B. Labelling of hypertensives: a review of the data. J Clin Epidemiol 1990;43:195-200. doi: 10.1016/0895-4356(90)90185-R pmid: 2406378

39 Barger SD, Muldoon MF. Hypertension labelling was associated with poorer self-rated health in the Third US National Health and Nutrition Examination Survey. J Hum Hypertens 2006;20:117-23. doi: 10.1038/sj.jhh.1001950 pmid: 16267563

40 Bell KJL, Doust J, Glasziou P. Incremental benefits and harms of the 2017 American College of Cardiology/American Heart Association high blood pressure guideline. JAMA Intern Med 2018;178:755-7. doi: 10.1001/jamainternmed.2018.0310 pmid: 29710197

41 Gyuricza JV, d'Oliveira AFPL, Machado LBM, Brodersen J. Development of an item pool for a questionnaire on the psychosocial consequences of hypertension labelling. J Patient Rep Outcomes 2019;4:2. doi: 10.1186/s41687-019-0168-4.. pmid: 31893320

42 Reventlow SD, Hvas L, Malterud K. Making the invisible body visible. Bone scans, osteoporosis and women's bodily experiences. Soc Sci Med 2006;62:2720-31. doi: 10.1016/j.socscimed.2005.11.009 pmid: 16356616

43 Martin SA, Boucher M, Wright JM, Saini V. Mild hypertension in people at low risk. BMJ 2014;349:g5432. doi: 10.1136/bmi.g5432 pmid: 25224509 